\title{
Controllable Kerr Nonlinearity in a Degenerate V- Type Inhomogeneously Broadening Atomic Medium Aided By a Magnetic Field
}

Dong Hoang Minh ( $\nabla$ dong.gtvtmt@gmail.com )

Ho Chi Minh City University of Food Industry https://orcid.org/0000-0003-0339-8192

Thanh Thai Doan

Ho Chi Minh City University of Food Industry

Anh Nguyen Tuan

Ho Chi Minh City University of Food Industry

\section{Research Article}

Keywords: magnetic field, atomic model, optical switching

Posted Date: October 21st, 2021

DOI: https://doi.org/10.21203/rs.3.rs-925076/v1

License: (c) (i) This work is licensed under a Creative Commons Attribution 4.0 International License.

Read Full License 


\title{
Controllable Kerr nonlinearity in a degenerate V-type inhomogeneously broadening atomic medium aided by a magnetic field
}

\author{
Hoang Minh Dong ${ }^{(*)}$, Thai Doan Thanh, and Nguyen Tuan Anh \\ Ho Chi Minh City University of Food Industry, Ho Chi Minh City, Vietnam \\ *Corresponding author. E-mail: dong.gtvtmt@gmail.com
}

\begin{abstract}
We propose a degenerate V-type inhomogeneously broadening atomic model for Kerr nonlinear control under the support of an external magnetic field. The magnetic field is used as a control knob to convert the medium from transparency to absorption at the resonance center. It is shown that the magnitude and sign of the Kerr nonlinearity index are manipulated by the magnetic field. Especially, we can easily convert the Kerr nonlinearity index from the positive peak into a negative peak, and from zero value can be transferred to enhanced positive or negative peaks simply only by changing the direction or strength of the magnetic field. In addition, the Kerr nonlinearity index under the influence of the temperature, the intensity, and detuning of the control field is also considered. The proposed model can be useful for applications in optical switching and nonlinear optical devices as well as possible experimental implementations.
\end{abstract}

\section{Introduction}

Kerr nonlinear control plays a very important role in nonlinear optics because it has many engaging applications in different fields [1-3]. For example, the balance between the Kerr nonlinear coefficient and group velocity dispersion in the medium is determining factor in the generation of optical solitons $[4,5]$. The small nonlinearity and passive optical properties of the traditional two-level atomic medium have limited their applicability in photonic devices. Recently, there has been a significant shift in the research interest of scientists to multi-level atomic systems related to quantum coherence and interference phenomena [6]. Typical among 
them is the discovery of the electromagnetically induced transparency (EIT) effect $[7,8]$, which has opened up many potential applications [9-17]. The EIT effect reduces the resonance absorption and increases the sharps dispersion, thereby notably increasing the interaction time between light and matter, leading to enhance nonlinear optical processes under conditions of low-light intensity or even single-photon levels [18-22].

Kerr nonlinear enhancement based on the EIT effect has been widely studied both experimentally and theoretically [23-34]. One of the pioneering works in this field was proposed by Schmidt and Imamoglu in 1996 [23]. It is shown that can be achieved a giant increase of Kerr nonlinearity in a four-level N-type atomic system under the EIT regime by introducing an additional off-resonant level. Then, this scheme was proved experimentally in cold $\mathrm{Rb}$ atoms by Kang and Zhu [24]. Furthermore, for the first time in the experiment, Wang et al. [26] measured the Kerr nonlinear index of the lambda-type three-level atomic medium at room temperature inside an optical ring cavity. This work has shown that the Kerr nonlinear index can be modified and significantly enhances according to the coherence parameters of the coupling laser fields. At the same time, the sign of the nonlinear index can be modified by changing the sign of the control beam frequency detuning. Sheng et al. [28] demonstrated that adjusting the switching laser power makes it possible to enhance the Kerr nonlinearity in the $\mathrm{N}$-type four-level system in the resonant neighborhood. Besides, using the double-dark resonant interaction in the four-level tripod-type atomic system such as large Kerr nonlinearity through cross-phase modulation [29, 30], store a light pulse in two channels [31] and obtain a subDoppler and subnatural narrowing of an absorption line [32], and via the incoherent pumping [34]. In addition, a series of studies to enhance Kerr nonlinearity under the influence of spontaneously generated coherence and relative phase between coupling laser fields have also been performed [36-42], such as colossal Kerr nonlinearity can be obtained in a V-type system under the influence of SGC [36], or the relative phase of the coupling fields [41]. 
Recently, the external magnetic field used as a control parameter has also attracted many studies such as the ultraslow light-dark soliton transition [43], pulse propagation and optical switching [44[, controlling the period and threshold intensity of the bistability [45-48]. However, using the external magnetic field to control Kerr nonlinearity in a V-type inhomogeneously broadening medium has not been studied. In this paper, the Kerr nonlinear index in a degenerated V-type atomic medium under the influence of Doppler broadening can be manipulated by a magnetic field. To facilitate this, we derive an analytic solution expression for the Kerr nonlinear index $n_{2}$ under Doppler broadening and the magnetic field. From there, we control the amplitude and sign of the Kerr nonlinear index under the influence of the magnetic field, intensity, and frequency detuning of the control field in the presence of Doppler broadening. The results obtained are useful for applications in magneto-optic switching and logic gates devices. Furthermore, the model is easy to realize in an experiment because the system can be performed in different frequency regimes using only a single laser for both coupling and probe fields.

\section{Model and basic equations}

The model of a degenerate V-type atomic system under the effect of an external magnetic field, and two probe and control laser fields are depicted in Fig. 1. Here, the transition $|2\rangle \leftrightarrow|1\rangle$ is coupled by the weak probe laser field Ep has the Rabi frequency $\Omega_{p}=\mu_{21} E_{p} / 2 \mathrm{~h}$ and the left-circularly polarized $\sigma^{-}$; whereas, the transition $|3\rangle \leftrightarrow|1\rangle$ is coupled with the strong coupling laser field $E_{c}$ with Rabi frequency $\Omega_{c}=\mu_{23} E_{c} / 2 \mathrm{~h}$ and the right-circularly polarized $\sigma^{+}$. The medium is subject to an applied longitudinal magnetic field B that removes the degeneracy of the states $|2\rangle$ and $|3\rangle$, whose Zeeman shift is determined by $\Delta_{B}=\mu_{B} m_{F} g_{F} B / \mathrm{h}$, where $\mu_{B}$ is the Bohr magneton, $g_{F}$ is the Lande factor, and $m_{F}= \pm 1$ is the magnetic quantum number of the corresponding state. Before interacting with atoms, the probe and control fields are directed towards a quarter-wave plate (QWP) to produce right- and left-circularly polarization 
components [44-46], respectively. The decay rates from the states $|3\rangle$ and $|2\rangle$ to $|1\rangle$ are given by $\gamma_{31}$ and $\gamma_{21}$, respectively.

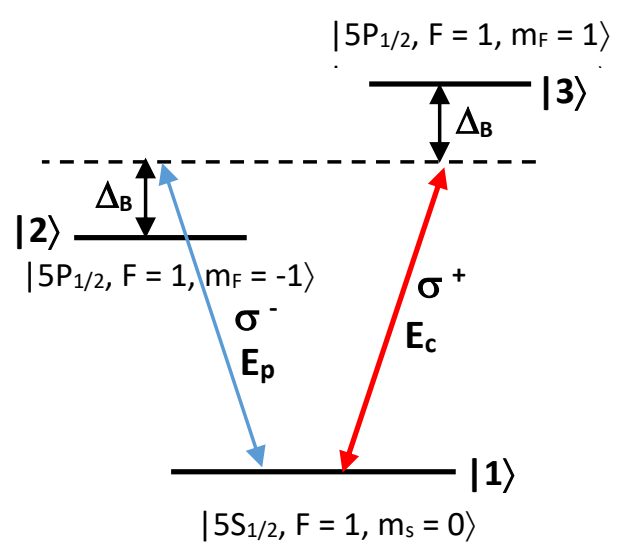

Fig. 1. The degenerate V-type atomic model interacting with control, probe, and external magnetic fields.

The total Hamiltonian in the interaction picture of the atomic and the laser field for this system is given by $[14,44]$ :

$$
H_{\text {int }}=-\left(\Delta_{p}+\Delta_{B}\right)|2\rangle\left\langle 2\left|+\left(\Delta_{B}-\Delta_{c}\right)\right| 3\right\rangle\langle 3|-\left(\Omega_{p}|2\rangle\left\langle 1\left|+\Omega_{c}\right| 3\right\rangle\langle 1|+H . c\right),
$$

here assume $\mathrm{h}=1$, and notation $\Delta_{c}=\omega_{31}-\omega_{c}$ and $\Delta_{p}=\omega_{21}-\omega_{p}$ are the frequency detuning of the coupling and probe fields, respectively.

The dynamic evolution of the system is described by the Liouville equation:

$$
\frac{\partial \rho}{\partial t}=-i\left[H_{\mathrm{int}}, \rho\right]+\Lambda \rho
$$

The density matrix equations for this system can be written as:

$$
\begin{aligned}
& \frac{\partial \rho_{11}}{\partial t}=\gamma_{21} \rho_{22}+\gamma_{31} \rho_{33}+i \Omega_{p}\left(\rho_{21}-\rho_{12}\right)+i \Omega_{c}\left(\rho_{31}-\rho_{13}\right), \\
& \frac{\partial \rho_{22}}{\partial t}=-\gamma_{21} \rho_{22}+i \Omega_{p}\left(\rho_{12}-\rho_{21}\right),
\end{aligned}
$$




$$
\begin{aligned}
& \frac{\partial \rho_{33}}{\partial t}=-\gamma_{31} \rho_{33}+i \Omega_{c}\left(\rho_{13}-\rho_{31}\right), \\
& \frac{\partial \rho_{21}}{\partial t}=-\left(\frac{\gamma_{21}}{2}-i\left(\Delta_{p}+\Delta_{B}\right)\right) \rho_{21}+i \Omega_{p}\left(\rho_{11}-\rho_{22}\right)-i \Omega_{c} \rho_{23}, \\
& \frac{\partial \rho_{31}}{\partial t}=-\left(\frac{\gamma_{31}}{2}-i\left(\Delta_{c}-\Delta_{B}\right)\right) \rho_{31}+i \Omega_{c}\left(\rho_{11}-\rho_{33}\right)-i \Omega_{p} \rho_{32}, \\
& \frac{\partial \rho_{23}}{\partial t}=-\left(\frac{\gamma}{2}-i\left(\Delta_{p}-\Delta_{c}+2 \Delta_{B}\right)\right) \rho_{23}+i \Omega_{p} \rho_{13}-i \Omega_{c} \rho_{21},
\end{aligned}
$$

where, $\rho_{i j}=\rho_{j i}^{*}(\mathrm{i}, \mathrm{j}=1,2,3 ; \mathrm{i} \neq \mathrm{j}), \rho_{11}+\rho_{22}+\rho_{33}=1 ; \gamma=\left(\gamma_{21}+\gamma_{31}\right)$, and $\gamma_{i j}$ is the decay rate between levels $|\mathrm{i}\rangle$ and $|\mathrm{j}\rangle$, respectively.

To obtain linear and nonlinear susceptibilities, we need to give the steady-state solution of the density matrix equations (3). To attain this aim, the perturbation approach is used to the density matrix elements are expressed as: $\rho_{m n}=\rho_{m n}^{(0)}+\rho_{m n}^{(1)}+\rho_{m n}^{(2)}+\rho_{m n}^{(3)}+\ldots \rho_{m n}^{(n)}$. We assume that the coupling field is much stronger than the probe field and the zeroth-order solution of the population $\rho_{11}^{(0)}=1$, while the other elements are equal to zero. In the weak-probe field approximation, we obtain the first-order and third-order solutions of the matrix element $\rho_{21}$ from equations (3d) and (3f):

$$
\rho_{21}^{(1)}=\frac{i \Omega_{p}\left(\rho_{11}^{(0)}-\rho_{22}^{(0)}\right)}{\gamma_{21} / 2-i\left(\Delta_{p}+\Delta_{B}\right)+\frac{\Omega_{c}^{2}}{\gamma / 2-i\left(\Delta_{p}-\Delta_{c}+2 \Delta_{B}\right)}} ; \frac{i \Omega_{p}}{M}
$$

and

$$
\rho_{21}^{(3)}=\frac{i \Omega_{p}\left(\rho_{11}^{(2)}-\rho_{22}^{(2)}\right)}{M},
$$

where

$$
M=\gamma_{21} / 2-i\left(\Delta_{p}+\Delta_{B}\right)+\frac{\Omega_{c}^{2}}{\gamma / 2-i\left(\Delta_{p}-\Delta_{c}+2 \Delta_{B}\right)} .
$$


To explicitly derive the element $\rho_{21}^{(3)}$, we need to calculate the population difference $\rho_{11}^{(2)}-\rho_{22}^{(2)}$. Assuming that $\rho_{33}^{(2)}=0$ and $\rho_{11}^{(2)}+\rho_{22}^{(2)}+\rho_{33}^{(2)}=0$, so that from equations (3a) and (3b), $\rho_{11}^{(2)}-\rho_{22}^{(2)}$ can be determined as follows:

$$
\rho_{11}^{(2)}-\rho_{22}^{(2)}=\frac{-2 i \Omega_{p}}{\gamma_{21}}\left(\rho_{12}^{(1)}-\rho_{21}^{(1)}\right)=\frac{-2 \Omega_{p}^{2}}{\gamma_{21}}\left(\frac{1}{M}+\frac{1}{M^{*}}\right) .
$$

Substituting Eq. (7) into Eq. (5), we obtain the element $\rho_{21}^{(3)}$ as follows:

$$
\rho_{21}^{(3)}=\frac{-2 i}{\gamma_{21}} \frac{\Omega_{p}^{3}}{M}\left(\frac{1}{M}+\frac{1}{M^{*}}\right)
$$

Therefore, the first- and third-order susceptibilities are determined as follows:

$$
\begin{aligned}
& \chi^{(1)}=\frac{N\left|\mu_{21}\right|^{2}}{\varepsilon_{0} \mathrm{~h} \Omega_{p}} \rho_{21}^{(1)}=\frac{i N\left|\mu_{21}\right|^{2}}{\varepsilon_{0} \mathrm{~h}} \frac{1}{M} \\
& \chi^{(3)}=\frac{N\left|\mu_{21}\right|^{2}}{3 \varepsilon_{0} \mathrm{~h} \Omega_{p}^{3}} \rho_{21}^{(3)}=-\frac{i N\left|\mu_{21}\right|^{4}}{6 \varepsilon_{0} \mathrm{~h}^{3} \gamma_{21}} \frac{1}{M}\left(\frac{1}{M}+\frac{1}{M^{*}}\right)
\end{aligned}
$$

where the total susceptibility $\chi=\chi^{(1)}+3 E_{p}^{2} \chi^{(3)}$ and $\mu_{21}$ is the dipole moment for the $|2\rangle \leftrightarrow|1\rangle$ transition.

The susceptibility expressions above do not include the effect of Dopper broadening. However, to match the experimental situations under room temperature conditions, we need to consider the Doppler broadening. Here the probe beam and the coupling beam are copropagating in the medium. Therefore, an atom moving towards the probe beam with velocity $\mathrm{v}$ will see the frequency of the probe beam up-shifted to $\omega_{p}+(v / c) \omega_{p}$, and that atom will see the frequency of the coupling beam up-shifted to $\omega_{c}+(v / c) \omega_{c}$. This lead to the frequency detuning of the laser beams will have modified as: $\Delta_{p}^{\prime}=\Delta_{p}+(v / c) \omega_{p}$ and $\Delta_{c}^{\prime}=\Delta_{c}+(v / c) \omega_{c}$. Assuming that the components of the atomic velocities which lie along the probe beam axis obey a Maxwellian distribution:

$$
d N(v)=\frac{N_{0}}{u \sqrt{\pi}} \exp \left(-\frac{v^{2}}{u^{2}}\right) d v
$$


where, $u=\sqrt{2 k_{B} T / m}$ is the root mean square atomic velocity and $N_{0}$ is the total atomic density of the vapour. In this way the Doppler effect can be included in the susceptibilities as:

$$
\begin{aligned}
& \chi^{(1)}(v) d v=\frac{i\left|\mu_{21}\right|^{2}}{\varepsilon_{0} \mathrm{~h}} \frac{1}{M(v)} d N(v), \\
& \chi^{(3)}(v) d v=-\frac{i\left|\mu_{21}\right|^{4}}{6 \varepsilon_{0} \mathrm{~h}^{3} \gamma_{21}} \frac{1}{M(v)}\left(\frac{1}{M(v)}+\frac{1}{M^{*}(v)}\right) d N(v),
\end{aligned}
$$

with

$$
M(v)=\gamma_{21} / 2-i\left(\Delta_{p}+\Delta_{B}(v / c) \omega_{p}\right)+\frac{\Omega_{c}^{2}}{\gamma / 2-i\left(\Delta_{p}-\Delta_{c}+2 \Delta_{B}\right)-i(v / c)\left(\omega_{p}-\omega_{c}\right)} .
$$

The first- and third-order susceptibility obtained by integrating equations (12) and (13) over the velocity $\mathrm{v}$ from $-\infty$ to $+\infty$, as:

$$
\begin{aligned}
\chi_{D}^{(1)}= & \frac{i N_{0}\left|\mu_{21}\right|^{2} \sqrt{\pi}}{\varepsilon_{0} \mathrm{~h}\left(\omega_{p} u / c\right)} e^{M_{D}^{2}}\left[1-\operatorname{erf}\left(M_{D}\right)\right], \\
\chi_{D}^{(3)}= & -\frac{i N_{0}\left|\mu_{21}\right|^{4}}{6 \sqrt{\pi} \varepsilon_{0} \mathrm{~h}^{3} \gamma_{21}\left(\omega_{p} u / c\right)^{2}} e^{M_{D}^{2}} \times\left\{2 \sqrt{\pi}\left(-1+\sqrt{\pi} M_{D} e^{M_{D}^{2}}\left[1-\operatorname{erf}\left(M_{D}\right)\right]\right)\right. \\
& \left.+\frac{\pi\left(e^{M_{D}^{2}}\left[1-\operatorname{erf}\left(M_{D}\right)\right]+e^{M_{D}^{2 *}}\left[1-\operatorname{erf}\left(M_{D}^{*}\right)\right]\right)}{M_{D}+M_{D}^{*}}\right\},
\end{aligned}
$$

where

$$
M_{D}=\frac{c}{\omega_{p} u}\left(\gamma_{21} / 2-i\left(\Delta_{p}+\Delta_{B}\right)+\frac{\Omega_{c}^{2}}{\gamma / 2-i\left(\Delta_{p}-\Delta_{c}+2 \Delta_{B}\right)}\right)=\frac{c}{\omega_{p} u} M
$$

and the $M_{D}{ }^{*}$ being complex conjugates of the $M_{D}$, and erf is the error function.

The linear index $\mathrm{n} 0$ and the Kerr-nonlinear index $\mathrm{n} 2$ coefficients are related to the first-order and third-order susceptibilities, $\chi^{(1)}$ and $\chi^{(3)}$, according to the relations [26]:

$$
\begin{aligned}
& n_{0}=\sqrt{1+\operatorname{Re}\left(\chi^{(1)}\right)}, \\
& n_{2}=\frac{3 \operatorname{Re}\left(\chi^{(3)}\right)}{4 \varepsilon_{0} n_{0}^{2} c} .
\end{aligned}
$$




\section{Results and discussions}

We apply the investigate model for the ${ }^{87} \mathrm{Rb}$ atomic medium with the levels $|2\rangle,|3\rangle$, and $|1\rangle$ chosened corresponding to states: $5 \mathrm{P}_{1 / 2}\left(F=1, m_{F}=-1\right), 5 \mathrm{P}_{1 / 2}\left(F=1, m_{F}=+1\right)$, and $5 \mathrm{~S}_{1 / 2}(F$ $\left.=1, m_{F}=0\right)$. The parameters are selected [26, 44]: $\gamma_{31}=\gamma_{21}=2 \pi \times 5.3 \mathrm{MHz}, N=4.5 \times 10^{17}$ atoms $/ \mathrm{m}^{3}, d_{21}=1.6 \times 10^{-29} \mathrm{C} . \mathrm{m}$, and the wavelength of the probe and control lasers is $\lambda_{p}=\lambda_{c}=$ $795 \mathrm{~nm}$. The Landé coefficient is $g_{F}=-1 / 2$ and the Bohr magnetron is $\mu_{B}=9.27401 \times 10^{-24} \mathrm{JT}^{-}$ ${ }^{1}[46,49]$. Here, the Zeeman shift $\Delta_{B}$ also calculated in units of $\gamma_{21}$, so the strength of the magnetic field $B$ will be given in units of the combined constant $\gamma_{c}=\hbar \gamma_{21} /\left(\mu_{B} g_{F}\right)$.

First, we consider the absorption and dispersion behavior of the probe field under the influence of an external magnetic field without and with the Doppler broadening effect, as shows in figures 2 and 3. Figs. 2(a) and (b), clearly shows that a magnetic field can be used to manipulate the responses of the medium for the probe field in the case no Doppler broadening. By varying the strength of the magnetic field it is possible to convert the medium from transparent to fully absorbent at the center of the EIT window [Fig. 2(a)]. At the same time, the normal dispersion lines are replaced by anomalous dispersion lines at the centerline with maximum absorption [Fig. 2(b)]. Namely, when the magnetic field has not been applied (i.e. $\mathrm{B}=0$ ), the collapses probe absorption at the resonance center corresponding to the EIT window is obtained at this position. Conversely, when the magnetic field is applied (i.e. $B \neq 0$ ), the probe absorption is enhanced at the center of the EIT window corresponding to the electromagnetically induced absorption (EIA). The EIT window is shifted to the left (or right) by an amount $\Delta_{p}=4 \gamma_{21}$ for $B=2 \gamma_{c}$, corresponds to $\Delta_{B}=2 \gamma_{21}$ (or $B=-2 \gamma_{c}$, corresponds to $\Delta_{B}=$ $-2 \gamma_{21}$ ), respectivelly. Physically, this EIT window displacement can be explained by the twophoton resonance condition $\left(\Delta_{p}-\Delta_{c}+2 \Delta_{B}=0\right)$ under the Zeeman splitting mode [46]. In the case with Doppler broadening, the obtained results are the same as the case non Doppler effect but have performance EIT is much smaller as depicted in Figs. 2(c) and (d). The physics reason 
here can be understood that under Doppler expansion (such as at room temperature), the $|2\rangle$ and |3) sublevels are overlapping. Therefore, to overcome this the magnetic field needs to be much stronger so that the Zeeman shift can be able to overcome this overlap. It is clear as described in Fig 3, where the coupling field is chosen $\Omega_{c}=80 \gamma_{21}$. From Fig 3(a), we can see the EIT window is shifted to the left (or right) by an amount $\Delta_{p}=120 \gamma_{21}$ for $B=60 \gamma_{c}$, corresponds to $\Delta_{B}=60 \gamma_{21}$ (or $B=-60 \gamma_{c}$, corresponds to $\Delta_{B}=-60 \gamma_{21}$ ), respectivelly. Therefore, the medium converting from transparency to fully absorbent at the resonance center and corresponding to the normal dispersion domain is also transferred to the anomalous dispersion domain and vice versa.
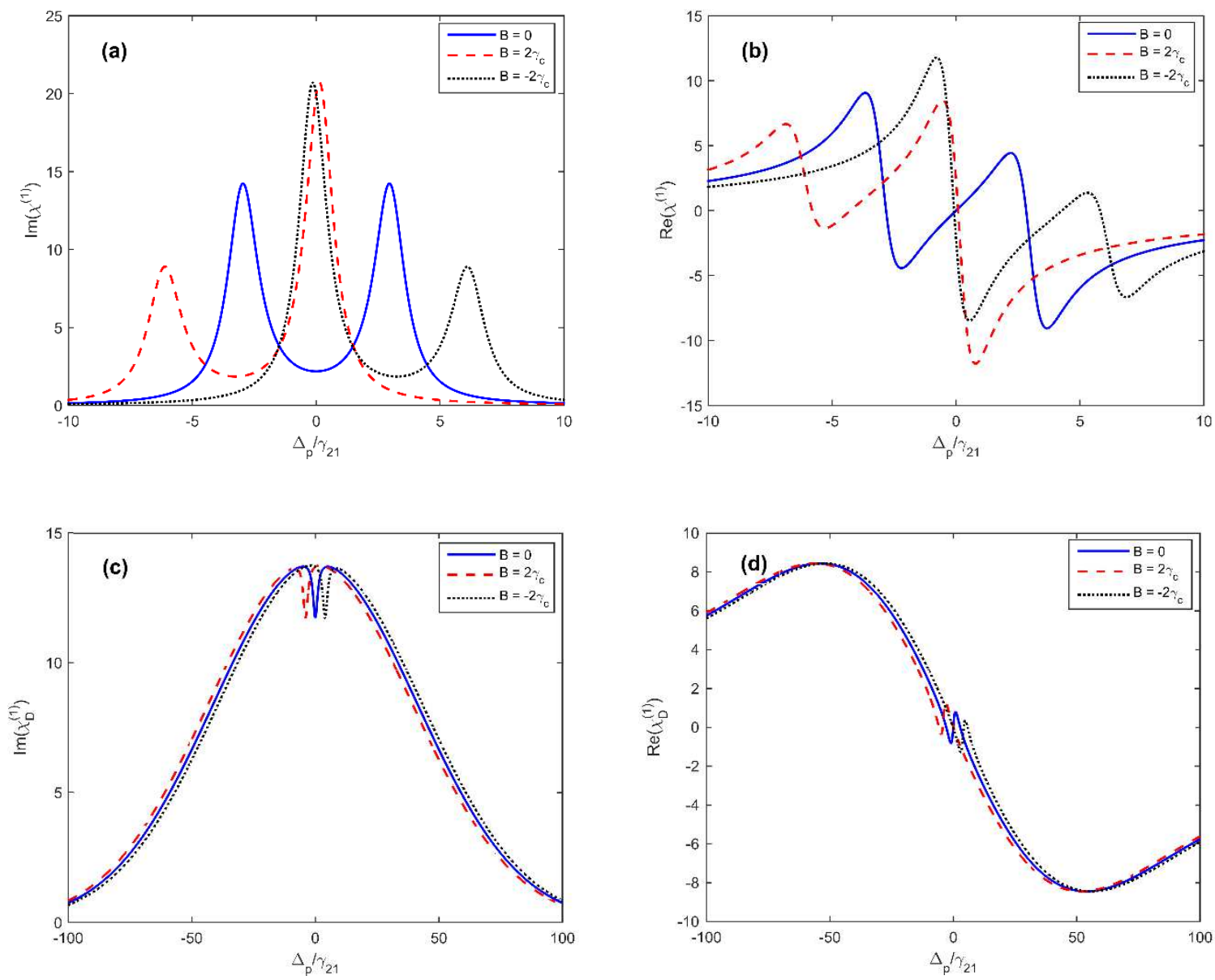

Fig. 2. Variations of the linear susceptibility $\chi_{D}^{(1)}$ versus the probe field detuning $\Delta_{p}$ for different values of the magnetic field $B$ without Doppler effect [panel (a) and (b)], and with Doppler 
effect [panel (c) and (d)]. The parameters are selected: $\Omega_{c}=3 \gamma_{21}, \Delta_{c}=0, \mathrm{~T}=300 \mathrm{~K}$, and $\gamma_{23}=\gamma_{21}$, respectively.
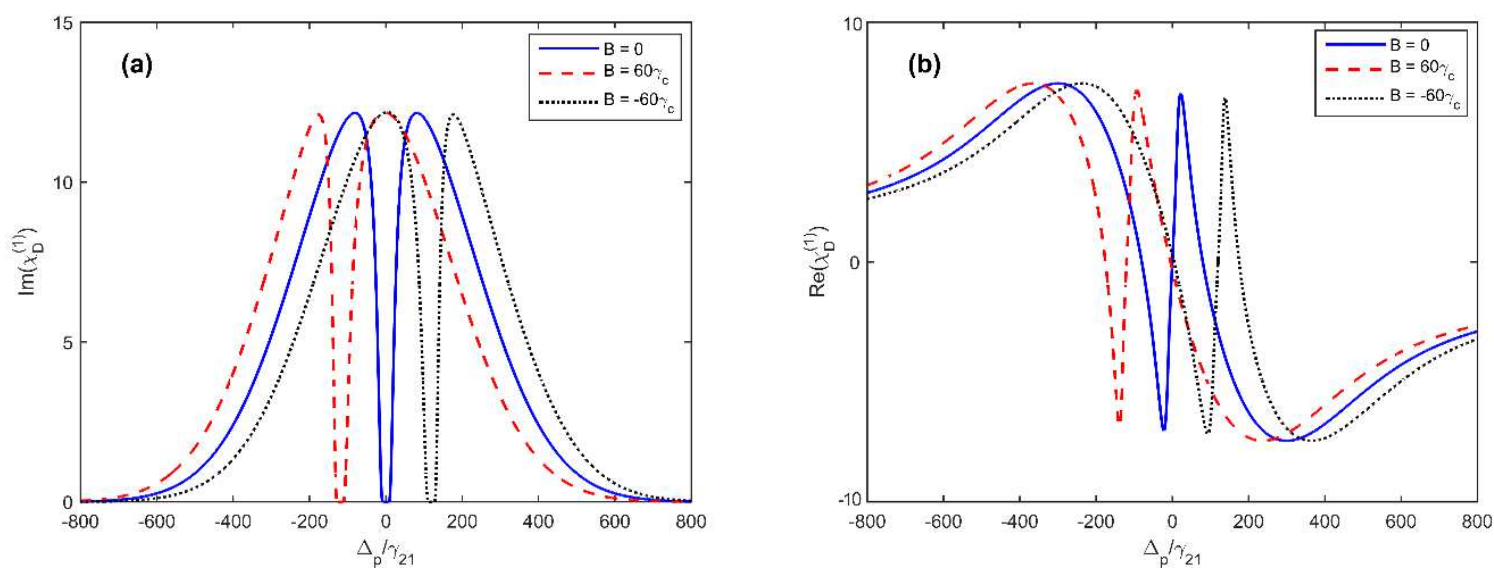

Fig. 3. Variations of the linear susceptibility $\chi_{D}^{(1)}$ versus the probe field detuning $\Delta_{p}$ for different values of the magnetic field $B$ with Doppler effect. The parameters are selected: $\Omega_{c}=80 \gamma_{21}$, $\Delta_{c}=0, \mathrm{~T}=300 \mathrm{~K}$, and $\gamma_{23}=\gamma_{21}$, respectively

Now, we will show that the external magnetic field can be used as a "knob" to manipulate the Kerr nonlinear index $n_{2}$. Fig. 4, shows that the amplitude and sign of the Kerr nonlinear index are dependent on the strength and direction of the magnetic field $B$. Specifically, in Fig. 4(a) for we can see that the positive peak of the Kerr nonlinear index at $\Delta_{p}$ $=-30 \gamma_{21}$ in the case of $B=0$ (solid line) has been shifted to the negative peak when $B=30 \gamma_{c}$ (dashed line). Conversely, the negative peak of the Kerr nonlinear index at $\Delta_{p}=30 \gamma_{21}$ in the case of $\mathrm{B}=0$ (solid line) has been shifted to the positive peak when $\mathrm{B}=-30 \gamma_{c}$ (dotted line). Such changes of the Kerr nonlinear index are similar to those obtained in [26, 27] for the case $\mathrm{B}=0$. Simultaneously, the frequency domains with a positive nonlinear index is also transferred to the frequency domains with a negative nonlinear index when switch-on/off of the magnetic field. In addition, the zero value of the Kerr nonlinear index at $\Delta_{p}=\Delta_{c}=0$ ) for the $\mathrm{B}=0$ [Fig. 4(b)] can be shifted to the negative peak when $B=15 \gamma_{c}$ (dashed line) or the positive peak when 
$\mathrm{B}=-15 \gamma_{c}$ (doded line). To gain a deeper understanding of this change, we plot the Kerr nonlinear index versus the magnetic field B as shown in Fig. 5. It is obvious to see that the Kerr nonlinear index is equal to zero at $\mathrm{B}=0$ has been transformed to the negative peak when $\mathrm{B}=$ $15 \gamma_{c}$ or the positive peak when $\mathrm{B}=-15 \gamma_{c}$. Thus, by selecting the appropriate magnetic field strengths and their direction, one can convert the Kerr nonlinear index from positive-negative or zero to negative-positive and vice versa. The Kerr nonlinear enhancement is achievable in the resonant frequency domain with absorption being suppressed. In Fig. 6, we represent the Kerr nonlinear index versus the coupling field detuning when $\Delta_{p}=0$. As shown in Fig. 6 , the negative peak of the Kerr nonlinear index at $\Delta_{c}=-30 \gamma_{21}$ or the positive peak at $\Delta_{c}=-30 \gamma_{21}$ in the case of $\mathrm{B}=0$ is shifted to the positive peak when $\mathrm{B}=-30 \gamma_{c}$ and the positive peak when $\mathrm{B}=$ $30 \gamma_{c}$. Furthermore, the zero value of the Kerr nonlinear index when $\mathrm{B}=0$ in the case of twophoton resonance has shifted to the negative peak when $\mathrm{B}=15 \gamma_{c}$ or the positive peak when $\mathrm{B}$ $=-15 \gamma_{c}$. This behavior can also be seen clearly as described in Fig. 4 .
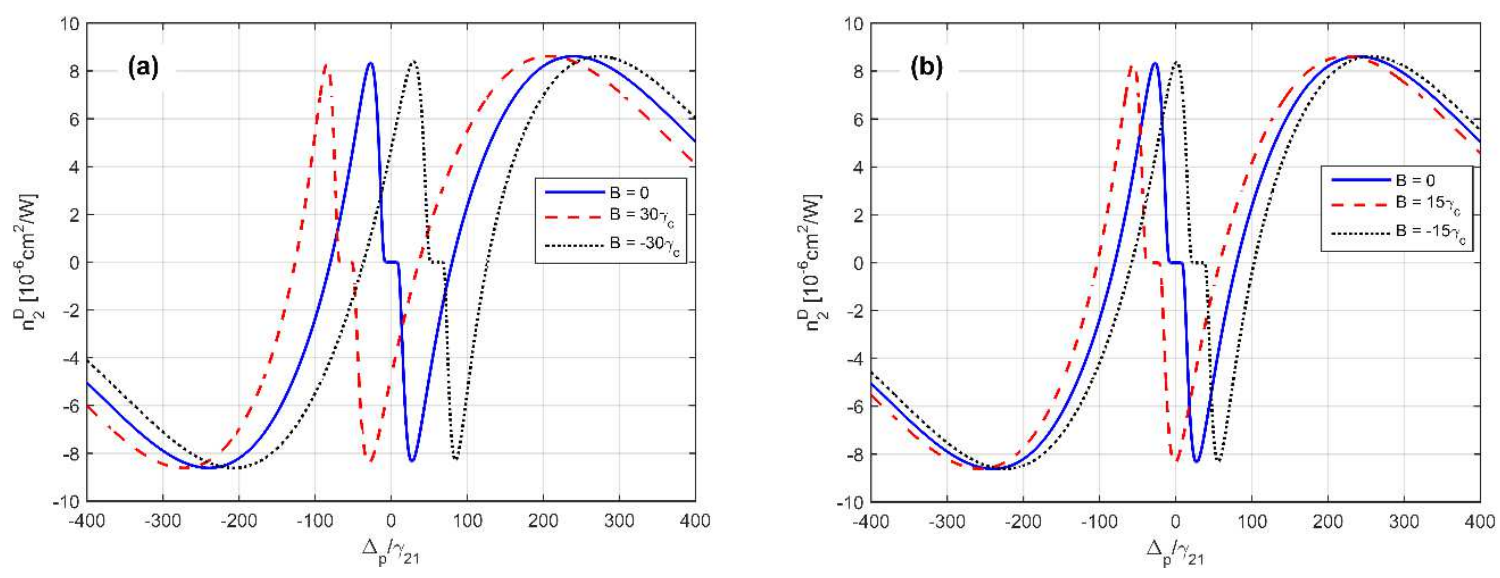

Fig. 4. Variations of the Kerr nonlinear index $n_{2}^{D}$ versus the probe field detuning $\Delta_{p}$ for the deffirent values of the magnetic field B in the presence Doppler broadening. The parameters are selected: $\Omega_{\mathrm{c}}=80 \gamma 21, \Delta_{c}=0, \mathrm{~T}=300 \mathrm{~K}$, and $\gamma_{23}=\gamma_{21}$, respectively. 


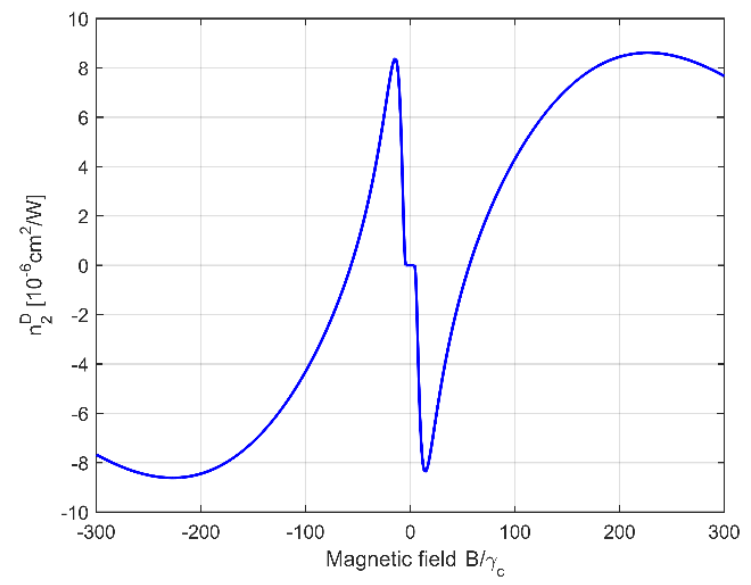

Fig. 5. The Kerr nonlinear index $n_{2}^{D}$ as function of the magnetic field $\mathrm{B}$. The parameters are selected: $\Omega_{\mathrm{c}}=80 \gamma_{21}, \Delta_{p}=\Delta_{c}=0, \mathrm{~T}=300 \mathrm{~K}$, and $\gamma_{23}=\gamma_{21}$, respectively.
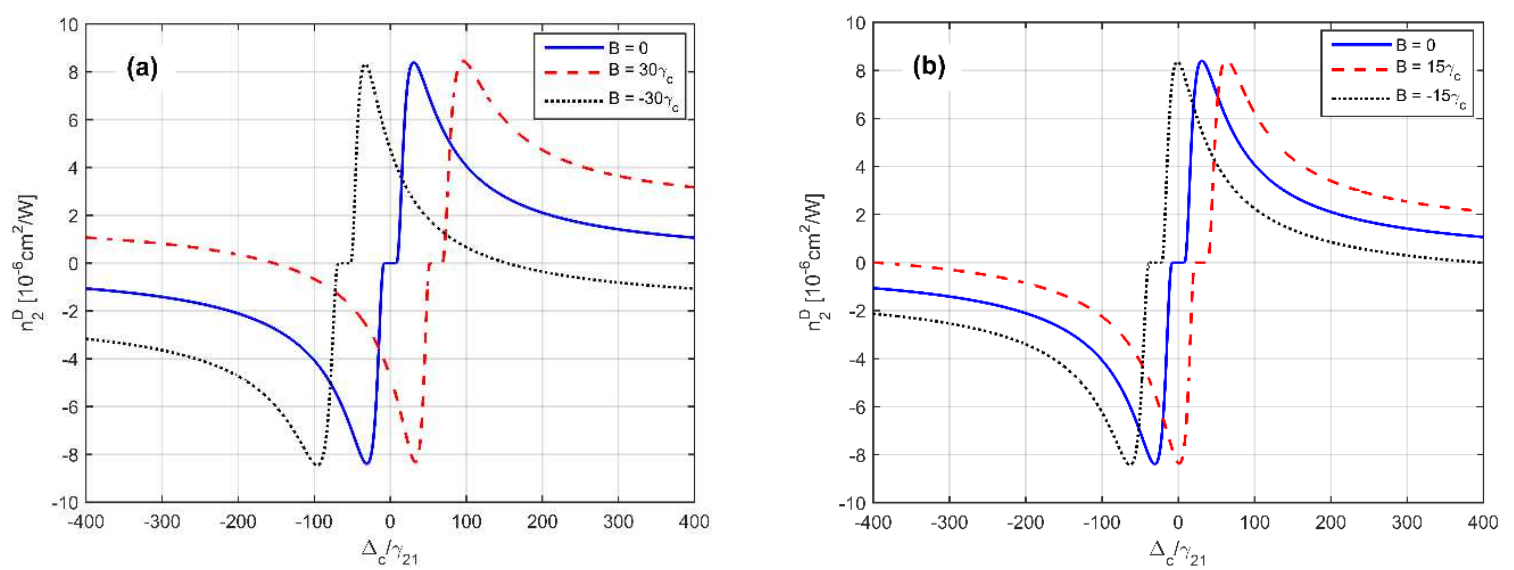

Fig. 6. Variations of the Kerr nonlinear index $n_{2}^{D}$ versus the coupling field detuning $\Delta_{c}$ for the deffirent values of the magnetic field B. Other parameters are the same as in Fig. 4, except $\Delta_{p}=0$.

Next, we investigate the variation of the Kerr nonlinear index according to the control laser field strength. As shown in Fig. 7, the amplitude and slope of the Kerr nonlinear index curve as a function of the intensity of the control beam. Indeed, in Fig. 7(a), we represent $n_{2}$ versus the $\Omega_{\mathrm{c}}$ when fixed $\Delta_{p}=30 \gamma_{21}$ which corresponds to the Kerr nonlinear index position with the largest amplitude in the two cases $B=0$ and $B=-30 \gamma_{c}$ [corresponding to Fig. 4(a)]. It shows that by adjusting the control beam intensity, one can also change the Kerr nonlinear index. Similarly, in Fig. 7(b) we plot for the two cases $B=15 \gamma_{c}$ and $B=-15 \gamma_{c}$ [corresponding 
to Fig. 4(b)] at the $\Delta_{p}=\Delta_{c}=0$. In particular, in this case, the Kerr nonlinear index variation is exactly symmetric over the zero-nonlinear dispersion line.
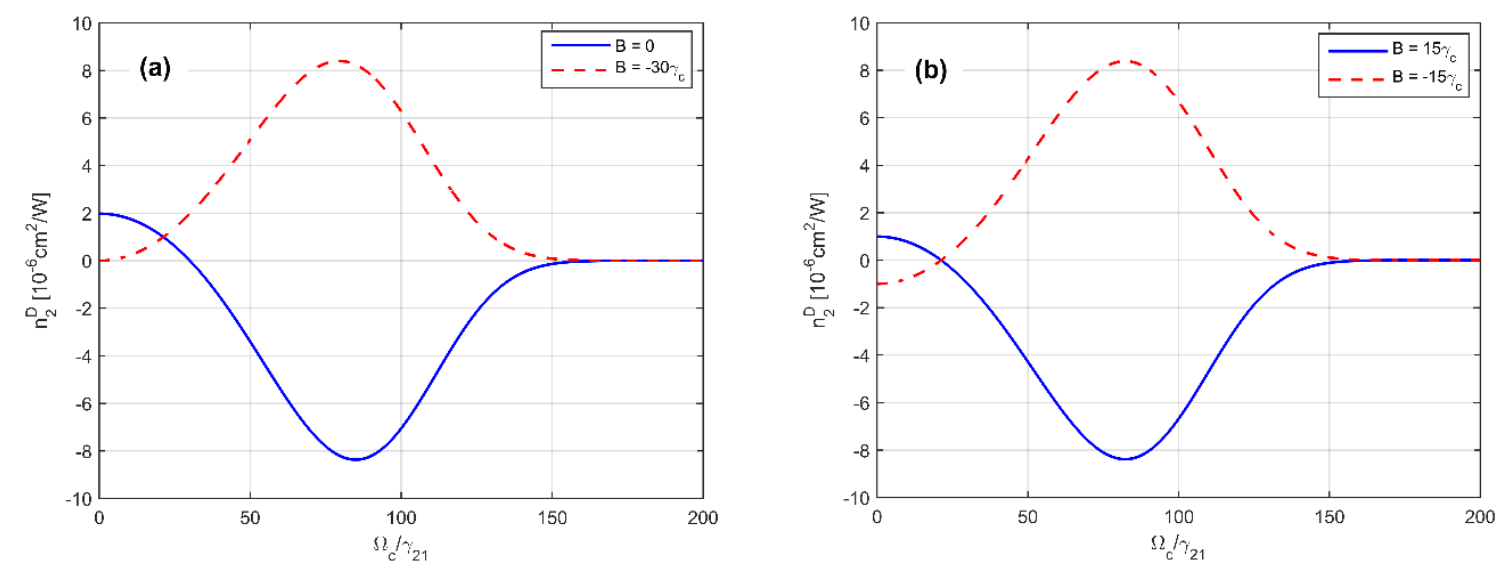

Fig. 7. The Kerr nonlinear index $n_{2}^{D}$ as function of the coupling field strength $\Omega_{\mathrm{c}}$ for the different values of magnetic field B. The parameters are selected: $\Delta_{p}=30 \gamma_{21}$, and $\Delta_{c}=0$ for (a), and $\Delta_{p}=\Delta_{c}=0$ for (b). Other parameters are the same as in Fig. 4.

Finally, we consider the influence of the Doppler broadening effect on the amplitude and shape of the Kerr nonlinear index curve $n_{2}$ as shown in Fig. 8. Fig. 8, shows the amplitude of the Kerr nonlinear index $n_{2}$ is degraded as the absolute temperature increases [27]. At the same time, the envelope of the nonlinear dispersion curve also gradually expands. The Kerr nonlinear index with controllable amplitude and sign has created many interesting applications in pulse propagation and all-optical switching technique.

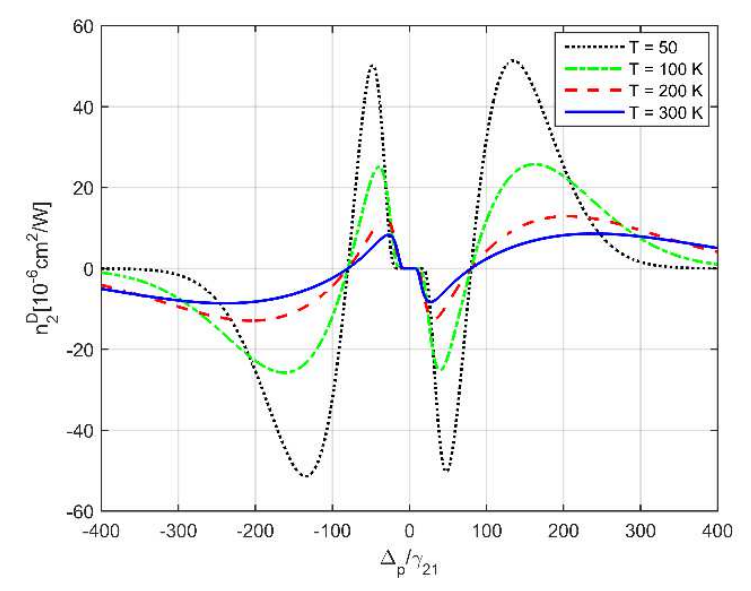


Fig. 8. Variations of the Kerr nonlinear index $n_{2}^{D}$ versus the probe field detuning $\Delta_{p}$ for the deffirent values of the absolute temperature: $\mathrm{T}=50 \mathrm{~K}$ (dotted line), $\mathrm{T}=100 \mathrm{~K}$ (dot-dashed line), $\mathrm{T}=200 \mathrm{~K}$ (dashed line), $\mathrm{T}=300 \mathrm{~K}$ (soild line). The parameters are selected: $\Omega_{\mathrm{c}}=80 \gamma_{21}$, $B=0$ or $\Delta_{B}=0, \Delta_{c}=0$, and $\gamma_{23}=\gamma_{21}$, respectively.

\section{Conclusions}

We have investigated the influence of external magnetic field on the Kerr nonlinearity index in a degenerate V-type atomic medium under the Doppler broadening and EIT effects. We have shown that the amplitude and sign of the Kerr nonlinear index are controlled by the external magnetic field, intensity, and frequency detuning of the control field. The result has been shown that by using the magnetic field as a control knob, we can convert the positive peak of the Kerr nonlinear index into the negative peak and vice versa. Moreover, it is possible to switch from the zero-nonlinear index into the largest value at the resonant center simply only by changing the direction and amplitude of the magnetic field. Manipulating the behavior of the Kerr nonlinear index is a favorable condition for generating steady propagating pulses (solitons) that have potential applications in all-optical switching and communications devices.

Declaration of Interest: The authors declare that they have no known competing financial interests or personal relationships that could have appeared to influence the work reported in this paper.

Acknowledgment: The study was supported by The Youth Incubator for Science and Technology Programe, managed by Youth Development Science and Technology Center - Ho Chi Minh Communist Youth Union and Department of Science and Technology of Ho Chi Minh City, the contract number is "01/2020/HĐ-KHCNT-VU". 


\section{References}

[1] H. A. Haus and W. S. Wong, "Solitons in optical communications", Rev. Mod. Phys., 68, No. 2, (1996).

[2] Joshi, M. Xiao, "Controlling Steady-State and Dynamical Properties of Atomic Optical Bistability”, World Scientific (2012).

[3] S. Rebic, D. Vitali, C. Ottaviani, P. Tombesi, M. Artoni, F. Cataliotti, R. Corbalan, Polarization phase gate with a tripod atomic system, Phys. Rev. A 70, 032317 (2004).

[4] Y. Wu and L. Deng, "Ultraslow bright and dark optical solitons in a cold three-state medium”, Opt. Lett. 29, 2064 (2004).

[5] Y. Chen, Z. Bai, and G. Huang, "Ultraslow optical solitons and their storage and retrieval in an ultracold ladder-type atomic system”, Phys. Rev. A 89, 023835 (2014).

[6] M. Fleischhauer, A. Imamoglu, J. P. Marangos, "Electromagnetically induced transparency: optics in coherent media”, Rev. Mod. Phys. 77, 633 (2005).

[7] K. J. Boller, A. Imamoglu, S. E Harris, "Observation of electromagnetically induced transparency”, Phys. Rev. Lett. 66, 2593 (1991).

[8] S. E. Harris, "Electromagnetically induced transparency", Phys. Today 50, 36 (1997).

[9] L. V. Hau, S. E. Harris, Z. Dutton, C. H. Bejroozi, "Light speed reduction to 17 meters per second in an ultracold atomic gas". Nature. 397, 594-598 (1999).

[10] T. D. Thanh, N. T. Anh, N. T. T. Hien, H. M. Dong, N. X. Hao, D. X. Khoa, N. H. Bang, "Subluminal and superluminal light pulse propagation under an external magnetic field in a vee-type three-level atomic medium”, Photonics Lett. Pol, 13 (1), 4-6 (2021).

[11] D. X. Khoa, L. V. Doai, D. H. Son, and N. H. Bang, "Enhancement of self-Kerr nonlinearity via electromagnetically induced transparency in a five-level cascade system: an analytical approach,” J. Opt. Soc. Am. B 31, 1330-1334 (2014). 
[12] H. M. Dong, L. V. Doai, V. N. Sau, D. X. Khoa and N. H. Bang, "Propagation of laser pulse in a three-level cascade atomic medium under conditions of electromagnetically induced transparency”, Photonics Lett. Pol, 8, 73 (2016).

[13] D. X. Khoa, H. M. Dong, L. V. Doai and N. H. Bang, "Propagation of laser pulse in a three-level cascade inhomogeneously broadened medium under electromagnetically induced transparency conditions", Optik 131, 497 (2017).

[14] H. M. Dong, L.V. Doai, and N. H. Bang, "Pulse propagation in an atomic medium under spontaneously generated coherence, incoherent pumping, and relative laser phase", Opt. Commun. 426, 553 (2018).

[15] H. M. Dong, and N. H. Bang, "Controllable optical switching in a closed-loop threelevel lambda system”, Phy. Scr. 94, 115510 (2019).

[16] N.T. Anh, T.D. Thanh, N.H. Bang, H.M. Dong, "Microwave assisted all-optical switching in a four-level atomic system”, Pramana - J. Phys. 95, 37 (2021).

[17] N. T. Anh, L. V. Doai, and N. H. Bang, "Manipulating multi-frequency light in a fivelevel cascade-type atomic medium associated with giant self-Kerr nonlinearity,” J. Opt. Soc. Am. B 35, 1233-1239 (2018).

[18] S. Sevinli, N. Henkel, C. Ates, T. Pohl, "Nonlocal nonlinear optics in cold Rydberg gases”, Phys. Rev. Lett. 107, 153001 (2011).

[19] T. Peyronel, O. Firstenberg, Q.Y. Liang, S. Hofferberth, A.V. Gorshkov, T. Pohl, M.D. Lukin, V. Vuletic, "Quantum nonlinear optics with single photons enabled by strongly interacting atoms", Nature 488, 57-60 (2012).

[20] O. Firstenberg, T. Peyronel, Q.Y. Liang, A.V. Gorshkov, M.D. Lukin, V. Vuletic, “Attractive photons in a quantum nonlinear medium”, Nature 502, 71-75 (2013).

[21] V. Venkataraman, K. Saha K and A. L. Gaeta, "Phase modulation at the few-photon level for weak-nonlinearity-based quantum computing", Nature Photon 7, 138-141 (2013). 
[22] D.E. Chang, V. Vuletic, M.D. Lukin, "Quantum nonlinear optics photon by photon", Nat. Photonics 8, 685-694 (2014).

[23] H. Schmidt and A. Imamoglu, "Giant Kerr nonlinearities obtained by electromagnetically induced transparency,” Opt. Lett. 21, 1936-1938 (1996).

[24] H. Kang, Y. Zhu, "Observation of large Kerr nonlinearity at low light intensities”, Phys. Rev. Lett. 91, 093601 (2003).

[25] T. Nakajima, "Enhanced nonlinearity and transparency via autoionizing resonance", Opt. Lett. $25,847-849$ (2000).

[26] H. Wang, D. Goorskey, M. Xiao, "Enhanced Kerr nonlinearity via atomic coherence in a three-level atomic system”, Phys. Rev. Lett. 87, 073601-1-4 (2001).

[27] L. V. Doai, D. X. Khoa and N. H. Bang, "EIT enhanced self-Kerr nonlinearity in the three-level lambda system under Doppler broadening”, Phys. Scr. 90, 045502 (2015).

[28] J. Sheng, X. Yang, H. Wu, and M. Xiao, "Modified self Kerr nonlinearity in a four-level N-type atomic system,” Phys. Rev. A 84, 053820 (2011).

[29] X.D. Yang, S.J. Li, C.H. Zhang, H. Wang, "Enhanced cross-Kerr nonlinearity via electromagnetically induced transparency in a four-level tripod atomic system”, J. Opt. Soc. Am. B 26, 1423-1434 (2009).

[30] Y. Han, J. Xiao, Y. Liu, C. Zhang, H. Wang, M. Xiao, and K. Peng, "Interacting dark states with enhanced nonlinearity in an ideal four-level tripod atomic system," Phys. Rev. A 77, 023824 (2008).

[31] Raczynski, M. Rzepecka, J. Zaremba, S.Z. Kaniasty, Polariton picture of light propagation and storing in a tripod system, Opt. Commun. 260, 73-80 (2006).

[32] Goren, A.D. Wilson-Gordon, M. Rosenbluh, H. Friedmann, Sub-Doppler and subnatural narrowing of an absorption line induced by interacting dark resonances in a tripod system, Phys. Rev. A 69, 063802 (2004). 

without absorption”, Phys. Lett. A 381, 3978-3982 (2017).

[34] H R Hamedi, A H Gharamaleki, and M Sahrai, "Colossal Kerr nonlinearity based on electromagnetically induced transparency in a five-level double-ladder atomic system", Appl. Opt. 22, 5892 (2016).

[35] N. H. Bang, D. X. Khoa, D. H. Son, and, L. V. Doai, "Effect of Doppler broadening on giant self-Kerr nonlinearity in a five-level ladder-type system,” J. Opt. Soc. Am. B 36(11), 3151-3158 (2019).

[36] Y. Niu and S. Gong, "Enhancing Kerr nonlinearity via spontaneously generated coherence,” Phys. Rev. A 73, 053811 (2006).

[37] X. A. Yan, L. Q. Wang, B. Y. Yin, W. J. Jiang, H. B. Zheng, J. P. Song, and Y. P. Zhang, "Effect of spontaneously generated coherence on Kerr nonlinearity in a four-level atomic system," Phys. Lett. A 372, 6456-6460 (2008).

[38] W. J. Jiang, J. P. Song, H. B. Zheng, C. Wu, B. Y. Yin, and Y. Zhang, "Enhancement of Kerr nonlinearity via spontaneously generated coherence in a four-level N-type atomic system,” Opt. Commun. 282, 101-105 (2009).

[39] X. Yan, L. Wang, B. Yin, and J. Song, "Enhanced self-Kerr nonlinearity in spontaneous emission”, J. Opt. Soc. Am. B 26, 1862-1868 (2009).

[40] S.H. Asadpour, M. Sahrai, A. Soltani, H.R. Hamedi, "Enhanced Kerr nonlinearity via quantum interference from spontaneous emission", Phys. Lett. A 376,147-152 (2012).

[41] H. Gao, H. Sun, S. Fan and H. Zhang, "Phase control of Kerr nonlinearity in V-type system with spontaneously generated coherence”, J. Mod. Opt. 63, 598-604 (2016).

[42] M. Sahrai and A. H. Gharamaleki, "Phase dependent Kerr nonlinearity via quantum interference”, Eur. Phys. J. D, 73: 129 (2019). 
[43] H.M. Dong, L.T.Y. Nga, D. X. Khoa, and N. H. Bang, "Controllable ultraslow optical solitons in a degenerated two-level atomic medium under EIT assisted by a magnetic field". Scientific Reports. 10 (1), 15298 (2020).

[44] J. Li, R. Yu, L. Si, and X. Yang, "Propagation of twin light pulses under magneto-optical switching operations in a four-level inverted-Y atomic medium”, J. Phys. B: At. Mol. Opt. Phys. 43, 065502 (2010).

[45] Z. Zhu, A. X. Chen, W. X. Yang, and R. K. Lee, "Phase knob for switching steady-state behaviors from bistability to multistability via spontaneously generated coherence", J. Opt. Soc. Am. B 31, 2061-2067 (2014).

[46] H. M. Dong, L. T. Y. Nga, N. H. Bang, "Optical switching and bistability in a degenerated two-level atomic medium under an external magnetic field”, Appl. Opt. 58(16), 4192-1499 (2019).

[47] Z. Kis, G. Demeter, and J. Janszky, "Pulse propagation in a dressed, degenerate system", J. Opt. Soc. Am. B 30, 829-837 (2013).

[48] P.J. Tsai, Y.C. Wei, B.H. Wu, S.X. Lin, and Y.C. Chen, "Theoretical study of a memory-based optical converter with degenerate Zeeman states”, Phys. Rev. A 100, 063843 (2019).

[49] D. A. Steck, (2019). 87Rb D line data, http://steck.us/alkalidata 